\title{
A simple Van der Heijde's Disease Activity Score to determine if a patient has contracted the new Nigerian variant or suffers from the Betcherew's syndrome
}

\section{Lorenzo Martini}

\author{
University of Siena, Department of Pharmaceutical Biotechnologies, Via A. Moro 2, 53100 Siena, Italy
}

Corresponding author: Prof. Lorenzo Martini, M.Sc., E-mail: lorenzo.martini @unisi.it

Sir,

A new strain of coronavirus first detected in Nigeria has made its way into the UK, with 32 cases reported. Eminent experts have warned the variant may prove resistant to all types of the vaccines.

Coronavirus has undergone yet another mutation and this one has made its way on to UK soil from Nigeria. There are growing fears that the variant will evade vaccine antibodies after it was revealed it contains the $484 \mathrm{~K}$ mutation to the spike protein. Last month, a Nigerian scientist suggested the variant may cause more severe signs and symptoms [1-3].

It has been affirmed that the variants discovered in the UK and South Africa, are distantly different from the variants discovered in Nigeria, and that clinically is the most severe as far as signs and symptoms. are concerned. Generally, severe signs and symptoms associated with COVID-19 include worsened shortness of breath and pneumonia. According to the health body, these symptoms may surface about a week after symptoms start.

In addition to the main symptoms of coronavirus, other symptoms include:

- Muscle aches

- Chills

- Sore throat

- Runny nose

- Headache

- Chest pain

- Pink eyes.
- Actually, Nigerian variant is more contagious than other mutations.

Nigerian variant is weaker than other mutations.

Nigerian variant is characterized by highest fever.

In Nigerian variant no fever is observed.

Nigerian variant is lethal to children.

Nigerian variant is lethal to elder.

Too many are informations about this newest covid strain that involves UK and Denmark, even if other countries that have entered at least one genome of this variant in the online database are Nigeria, the US, France, Canada, Ghana, Australia, Japan, Italy, Netherlands, Jordan, Singapore, Finland, Switzerland, Mayotte, Belgium and Spain.

While there is no information on what this means for this genome there is growing evidence that it may impact how effective COVID vaccines are. But there is no suggestion so far that Nigerian variant is more transmissible or that it leads to more severe disease.

Presently, the chief indicator to detect the assault by this odd and perilous variant is the presence of Pink eyes.

In this specific case, pink eyes are not sign of simplest conjunctivitis (both viral and bacterial) or allergis. It resembles the phenomenon of Pink eyes evoked by Sjoergen's syndrom, the extreme step of rheumatoid arthritis, as the Betchereff's syndrom in young athletes $[3,4]$.

We can affirm that a simplest Das 28 could be resolutive to understand if the patient has contracted the Nigerian

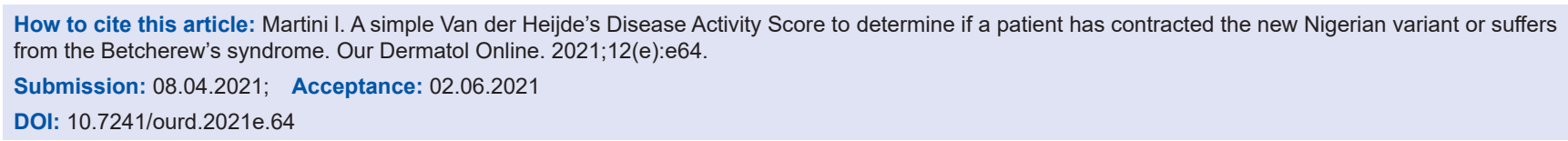


variant or proceeds with his own R.S., fully unaware. Das 28 is very easy to detect: one need only to appreciate the values of his own INR and OSR (erythrocyte sedimentation rate) and may find the module in the web, where one can insert these results and signal which of the 28 joints are really painful. The score is comprised between nihil and 5: the highest is the value the major is the chance to have pink eyes because of the R.A. and not absolutely because of the Nigerian variant.

\section{REFERENCES}

1. Nwakpu ES, Ezema VO, Ogbodo JN. Nigeria media framing of coronavirus pandemic and audience response. Health Promot
Perspect. 2020;10:192-9.

2. Amzat J, Aminu K, Kolo VI, Akinyele AA, Ogundairo JA, Danjibo MC. Coronavirus outbreak in Nigeria: Burden and socio-medical response during the first 100 days. Int J Infect Dis. 2020;98:218-24.

3. Ohia C, Bakarey AS, Ahmad T. COVID-19 and Nigeria: putting the realities in context. Int J Infect Dis. 2020;95:279-81.

4. Sigha OB, Kouotou EA. [COVID-19 infection revealed by a flare-up of psoriasis in an elderly Cameroonian: about a case]. Our Dermatol Online. 2021;12(Supp. 1):16-20.

Copyright by Lorenzo Martini. This is an open access article distributed under the terms of the Creative Commons Attribution License, which permits unrestricted use, distribution, and reproduction in any medium, provided the original author and source are credited.

Source of Support: Nil, Conflict of Interest: None declared. 